\title{
Las uniones infantiles forzadas: de la reforma legal a la disrupción social-Investigación formativa en cinco comunidades en Chisec Alta Verapaz
}

\author{
Paola Broll \\ Population Council \\ Cecilia Garcés \\ Population Council
}

Follow this and additional works at: https://knowledgecommons.popcouncil.org/departments_sbsr-pgy

Part of the Demography, Population, and Ecology Commons, Family, Life Course, and Society Commons, Gender and Sexuality Commons, International Public Health Commons, and the Maternal and Child Health Commons

How does access to this work benefit you? Let us know!

\section{Recommended Citation}

Broll, Paola and Cecilia Garcés. 2018. "Las uniones infantiles forzadas: de la reforma legal a la disrupción social-Investigación formativa en cinco comunidades en Chisec Alta Verapaz." Guatemala City:

Population Council. 
LAS UNIONES INFANTILES FORZADAS: DE LA REFORMA LEGAL A LA DISRUPCIÓN SOCIAL INVESTIGACIÓN FORMATIVA EN CINCO COMUNIDADES EN CHISEC ALTA VERAPAZ 


\section{population
councul \\ Ideas. Evidence. Impact.}

El Population Council enfrenta temas críticos en salud y desarrollo-desde detener la propagación del VIH hasta mejorar la salud reproductiva y asegurar que la gente joven disfrute una vida plena y productiva. A través de la investigación biomédica, en ciencias sociales y salud pública en 50 países, trabajamos con nuestros socios para ofrecer soluciones que conlleven a políticas públicas, programas y tecnologías más eficaces que mejoren la vida de las personas a nivel global. Fundado en 1952 y con sede en Nueva York, el Population Council es una organización no gubernamental regida por una junta directiva internacional.

Population Council - Guatemala Office

19 Avenida 0-35

Zona 15, Vista Hermosa 2

Ciudad de Guatemala

01015

email: info.guatemala@popcouncil.org

popcouncil.org

Cita sugerida: Broll, Paola and Cecilia Garcés. 2018. "Las uniones infantiles forzadas: de la reforma legal a la disrupción social-Investigación formativa en cinco comunidades en Chisec Alta Verapaz." Guatemala City: Population Council.

Equipo de investigación de campo: Julia Silvestre, Arturo Chub, Dinora Yat, Elvia Coc, Matilde Choc, Juana Pop, Juana Toj, Elva Tzoc, Modesta Ical, Susana Mo, Angustia Caal, Alicia Mo.

Revisoras: Alejandra Colom. Directora de País. Population Council. Walda Barrios-Klee. Coordinadora del Programa de Estudios de Género y Feminismos. Facultad Latinoamericana de Ciencias Sociales. Sede Académica de Guatemala.

(C) 2018 The Population Council, Inc. 


\section{Tabla de Contenido}

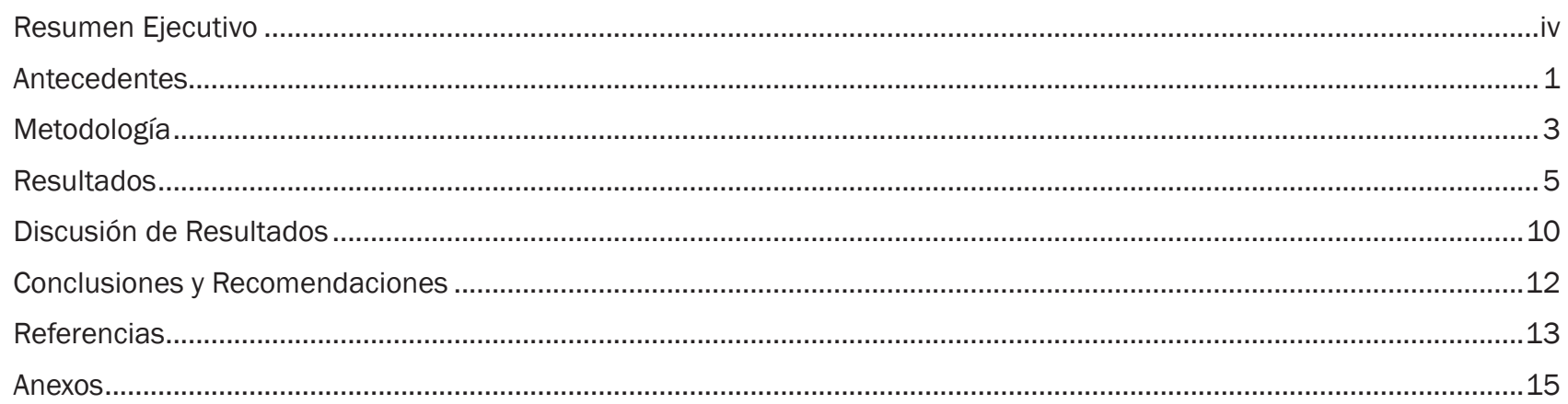




\section{Resumen Ejecutivo}

La evidencia ha demostrado que el matrimonio y las uniones infantiles forzadas (M/UIF) son nocivos a los derechos y el desarrollo de las niñas y adolescentes. También se ha demostrado que aumentar la edad mínima del matrimonio no es suficiente para erradicar este fenómeno que subyace en la práctica de las instituciones sociales a nivel comunitario. Realizamos esta investigación en el momento en que se aprobó la modificación al Código Civil 8-2015 seguido de la modificación 13-2017para aumentar la edad mínima para el matrimonio en Guatemala, con el propósito de identificar las normas, prácticas y actitudes vigentes en la escuela, la familia, la religión, el gobierno y la economía en relación con el M/UIF.

Se realizó una investigación formativa de carácter cualitativa en cinco comunidades de Chisec, Alta Verapaz, en donde se ha implementado el Programa Abriendo Oportunidades ${ }^{\circledR}$ por Population Council. Mediante historias de vida, encuestas a profundidad y grupos focales en las que participaron representantes de familias, iglesias, servicios de salud, escuelas y consejos comunitarios de desarrollo, se recolectó información sobre las prácticas que se dan en torno al M/UIF, lo que se conoce sobre su prohibición legal, y cuáles se consideran las causas y las consecuencias que tiene en la vida de las niñas y adolescentes. Además, se identificaron cambios en la práctica de los matrimonios y uniones tempranas pudiendo tipificar tres patrones en los que se presentan actualmente.

Con la información recopilada se diseñó y realizó una campaña radial en la región. Se crearon y difundieron mensajes dirigidos a padres y madres de familia, autoridades locales, escuelas e iglesias para dar a conocer la legislación y las consecuencias negativas para las niñas y adolescentes, con el fin de prevenir las uniones infantiles forzadas desde las instituciones sociales.

Se evidenció que existe información sobre la prohibición legal; así como las consecuencias negativas en la vida de las niñas y adolescentes. La posición de las familias y otros integrantes de la comunidad varía en torno al tema; sin embargo, se reconoce que aún se realizan uniones con apoyo de las familias, líderes religiosos y algunos representantes del gobierno. Un efecto interesante de la prohibición legal se documenta en la modificación del tratamiento que dan comadronas y otros proveedores de salud a niñas y adolescentes embarazadas, quienes ahora prefieren referirlas al sistema oficial de salud por las implicaciones legales tiene atender a menores de edad. Se identificaron tres patrones asociados al proceso de uniones: (1) En el pasado con los padres que arreglaban los matrimonios de las hijas sin que ellas pudieran opinar nada; (2) En el presente los padres que arreglan los matrimonios o uniones para solventar temas económicos o para ocultar un embarazo; y (3) los matrimonio o uniones decididos por las jóvenes. En el discurso generalizado se responsabiliza a las adolescentes de ser quienes mantienen el interés de unirse tempranamente para alcanzar mejores espacios de convivencia emocional y bienestar económico. Los cambios que se han dado entre una generación y otra, como al acceso a la escuela secundaria y a la tecnología, presenta nuevos retos en torno a los procesos de socialización de las adolescentes que deben ser atendidos mediante la implementación de una estrategia efectiva de educación integral en sexualidad.

Se concluye que la reforma a la ley sobre la edad mínima para contraer matrimonio ha provocado algún cambio a la práctica del matrimonio infantil; pero sus alcances son limitados. Esta investigación recomienda acciones que provoquen disrupción social a las prácticas y normas que tocan a las niñas y adolescentes desde todas las instituciones sociales. 


\section{Antecedentes}

Se conoce como matrimonio y la unión infantil forzada -M/ UIF- a cualquier matrimonio civil o unión de hecho en el que una o ambas partes son menores de 18 años (Naciones Unidas, s.f.). Alrededor del mundo, cada año, 15 millones de niñas son unidas/casadas antes de tener 18 años. Esto equivale a 28 niñas cada minuto y una cada dos segundos ${ }^{31}$. El M/UIF se reconoce como un problema en todo el mundo debido a sus efectos adversos en el acceso de las niñas a sus derechos y bienestar, ya que marca el final de su niñez, forzándolas a desempeñar actividades para las que no están física ni emocionalmente preparadas. ${ }^{2}$

Casarse antes de los 18 años se asocia con un aumento de la fecundidad y el crecimiento de la población, porque el matrimonio temprano alarga el tiempo que las niñas $y$ las mujeres permanecen en la edad fértil y acorta el lapso entre generaciones ${ }^{6}$. Esta práctica también socava la capacidad de una niña para acceder a la educación que permita desarrollar su autonomía y la expone a las consecuencias peligrosas del parto antes de la madurez física ${ }^{17}$. Las mujeres que se casan a edades más tempranas tienden a tener una mayor diferencia de edad con sus maridos, así como menor poder y autonomía en sus relaciones ${ }^{13,15}$ y en consecuencia tienen mayor riesgo de sufrir violencia doméstica. ${ }^{26,6,28,23}$

Population Council ha estudiado los efectos negativos del M/UIF y las condiciones de vida asociadas a su práctica con el fin de entender las instituciones sociales que lo legitiman y mantienen ${ }^{6}$. Estos estudios tienden a resaltar la relevancia de la educación, la pobreza, la religión y las tradiciones como factores asociados. La prevalencia de M/UIF tiende a persistir en áreas remotas, rurales y pobres ${ }^{6}$. Las mujeres con mayor probabilidad de casarse como niñas son aquellas que viven en áreas rurales, provienen de hogares pobres, se encuentra en contexto de crisis humanitaria y tienen poca o ninguna educación ${ }^{16,33}$. Es más probable que el M/UIF sea menor entre las mujeres que asistieron a la escuela secundaria que entre las que $\mathrm{no}^{28}$. En situaciones de pobreza, las familias dependen del M/UIF y la migración como estrategias para la supervivencia económica ${ }^{23}$.

Se sabe que retrasar el matrimonio puede mejorar las oportunidades de salud y productividad de las niñas y que estos beneficios se extienden a sus hijos, a su familia, a su comunidad y a su país ${ }^{1}$. Debido a los efectos nocivos del M/ UIF, su eliminación se ha convertido en una prioridad mayor en la agenda global y de varios países. El objetivo 5 de los Objetivos de Desarrollo Sostenible exige la erradicación del matrimonio infantil ${ }^{2}$. Las leyes claras y consistentes que prohíben el M/UIF se reconocen como un primer paso importante hacia su erradicación ${ }^{28}$; sin embargo, persisten las preguntas sobre la efectividad de esta estrategia. Al igual que en otros países donde se practica el M/UIF, Guatemala reformó el Código Civil en noviembre de $2015^{3}$ elevando la edad legal (18 años) para contraer matrimonio. Esta reforma aún contemplaba excepciones para poder contraer matrimonio a los 16 años, con autorización de un Juez. El 17 de agosto de 2017 se realizó otra reforma al Código Civil en la que se eliminaron todas las excepciones para contraer matrimonio antes de los 18 años ${ }^{4}$ (Congreso de la República de Guatemala, 2017). Este cambio, desde la institución del Estado para incidir sobre las prácticas y normas que rodean a la población, es relevante entre las políticas públicas y es el primer paso para su erradicación; sin embargo, Guatemala enfrenta aún dificultades con respecto a la aplicación de la ley pues ésta no es suficiente si no se modifican las circunstancias que determinan la exposición de las niñas a las uniones infantiles forzadas UIF. Aún con la ley apropiada, la práctica persiste por una variedad de razones, complejas e interdependientes. Los hombres manejan el dominio del poder en la mayoría de los aspectos de la vida restringiendo los derechos a las mujeres y las niñas, negándoles el ejercicio de la igualdad en su casa y en su comunidad. El manejo desigual se reproduce a través de las normas de género que otorgan mayor valor a los niños y hombres que a las mujeres ${ }^{16}$.

De las instituciones sociales emanan prácticas, normas y leyes que colocan a ciertos grupos de personas en desventaja, forzando su vulnerabilidad y creando barreras para su bienestar ${ }^{8}$. Las MUIF son ejemplo de ello, pues se ven influenciadas por las normas de la comunidad, la pobreza de los hogares y la falta de oportunidades ${ }^{1}$, todas las cuales se producen en el marco de las instituciones sociales. Colocar la mirada sobre las instituciones sociales permite analizar el contexto que rodea el MUIF, observar las prácticas sociales en las que se envuelve el fenómeno 
e identificar aquellas que colocan en vulnerabilidad a las niñas y adolescentes.

Si bien se ha generado una amplia gama de definiciones sobre las instituciones sociales, en este estudio las entendemos como el conjunto complejo, integrado y perdurable de normas, leyes y prácticas que buscan preservar las necesidades y valores sociales básicos de un grupo humano (Harper, Jones, \& Watson, 2012). Se reconocen cinco dimensiones amplias -meta instituciones- en las que operan las instituciones sociales: familia, gobierno y política, economía, educación y la religión. ${ }^{10}$

Estas dimensiones afectan toda la trayectoria de vida de las niñas y mujeres, y establecen los parámetros amplios dentro de los que coexisten formas particulares de normas, valores y prácticas. Es decir, la familia no es homogénea y estática y sus formas y dinámicas varían según el contexto ${ }^{10}$. Si bien son prácticas sociales recurrentes que perduran en el tiempo, también son susceptibles al cambio a partir de contradicciones internas o a la luz de modificaciones en el contexto, desde otras instituciones sociales ${ }^{10}$. Es fundamental conocer las recompensas y sanciones que experimentan familias, niñas y adolescentes cuando, desde las instituciones sociales, reproducen, sostienen o modifican las prácticas vinculadas al UIF². Este marco de análisis aborda también el vínculo existente entre normas, prácticas y la percepción de identidad grupal por la que diversos actores, incluidas las propias niñas y adolescentes, reproducen de forma tácita o explícita su apoyo a los M/UIF.

En Guatemala los datos existentes sobre el problema son muy limitados y provienen principalmente de las estadísti-

DIAGRAMA 1. Las cinco dimensiones en las que operan las instituciones sociales: familia, gobierno y política, economía, educación y la religión
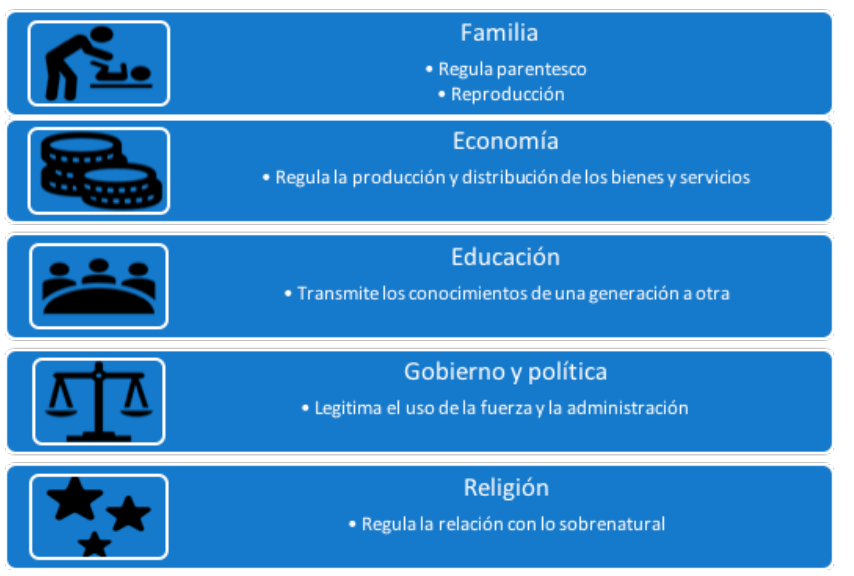

Elaboración propia con base al modelo de las dimensiones propuesto por Harper, Jones \& Watson. cas nacionales y la información asociada con los embarazos de adolescentes. Las estadísticas oficiales más recientes sobre M/UIF a nivel nacional son de 2009, y la prevalencia del matrimonio infantil se registra en el $7.7 \%$ de las niñas de 15 a 19 años casadas ${ }^{18}$.

Como en otras partes del mundo, en Guatemala el M/UIF se ha practicado durante generaciones. Algunas comunidades lo consideran una forma de proteger la honra de las niñas y las familias ante la posibilidad de las relaciones sexuales prematrimoniales ${ }^{17}$. El deseo de escapar de la pobreza y la violencia doméstica, exacerbado por la presión social para no convertirse en una "solterona", perpetúa el M/UIF como una práctica aceptada por la comunidad ${ }^{23}$. Un estudio en Chisec, Guatemala, determina que si bien las niñas tienen creciente participación en el proceso de toma de la decisión de realizar el M/UIF. Su elección debe estar en sintonía con las preferencias de su familia y comunidad, las políticas de gobierno y normas legales, oportunidades de educación y empleo ${ }^{32}$.

Al momento no se tienen evaluaciones sobre qué enfoques de intervención funcionan mejor para eliminar en M/UIF en Guatemala. Los principales estudios se enfocan en el embarazo adolescente, describen la metodología de las intervenciones para limitar el M/UIF, analizan su vínculo con la explotación sexual y la agencia de las niñas. Es necesario contar con evidencia local para el diseño de estrategias acertadas para la erradicación de este fenómeno.

Esta investigación explora las prácticas, normas y percepciones en torno al M/UIF de las y los representantes de diversas instituciones sociales en el nivel comunitario. El gobierno se aborda a través de las autoridades comunitarias, los proveedores de salud, la influencia de la municipalidad, los juzgados y el Registro Nacional de las Personas -RENAP-. El rol de la educación se evidencia a través de la acción o inacción de la escuela. La economía se analiza en la transacción latente en esta práctica. De las familias se obtienen las expectativas y normas en torno a la reproducción, mismas que -en muchos casos- son dictadas a través de la religión, aduciendo un carácter moral y sagrado. Identificar las instituciones sociales que incurren en producir, sostener y reproducir las vulnerabilidades de las niñas y adolescentes, busca comprender como éstas se construyen y cómo se pueden limitar ${ }^{10}$. La información revelada por esta investigación permitirá el diseño de acciones informativas dirigidas a actores de diversas instituciones sociales con el propósito de provocar la modificación de aquellas prácticas, normas y leyes que validan el M/UIF. 


\section{Metodología}

Esta investigación se planteó en el año 2016, en el marco del Decreto 08-2015, que aumentó la edad para contraer matrimonio a la mayoría de edad, con el propósito de explorar las prácticas, normas y percepciones que sobre el fenómeno del M/UIF tenían representantes de instituciones sociales en cinco comunidades del municipio de Chisec, Alta Verapaz ${ }^{a}$ en el momento que se realizaba la modificación a la edad mínima del matrimonio. Con fundamento en el marco conceptual del modelo ecológico que emplea Population Council , se diseñó la metodología e instrumentos de investigación. La metodología e instrumentos de la investigación fue presentada al Comité de Revisión Institucional (Institutional Review Board IRB) del Population Council y recibió una exención a una revisión completa pues no ponía en ningún riesgo a los adultos que participaron en la investigación.

Con el apoyo de participantes clave en cada una de las cinco comunidades, el equipo de investigación de campo -conformado por dos expertos investigadores sociales y diez mentoras capacitadas en el proceso de investigación social con énfasis en derechos humanos- recolectaron la información, en cuenta boletas con datos socioeconómicos de las personas consultadas para la investigación. Durante los meses de septiembre a diciembre de 2016 se realizaron grupos focales, historias de vida y entrevistas a profundidad con comadronas, lideresas, fundadores de la comunidad, padres y madres de familia, docentes, autoridades municipales y autoridades comunitarias. Se implementaron los instrumentos en el idioma materno de las y los participantes, en todos los casos se recogieron consentimientos informados documentando la participación libre y voluntaria, así como la autorización para el uso de la información obtenida.

La información recopilada fue transcrita, traducida al español ${ }^{c}$ y sistematizada en matrices de vaciado cualitativo, según instrumento de investigación y sujeto participante.

CUADRO 1. Técnicas, participantes e información recopilada

\begin{tabular}{|l|l|l|}
\hline \multicolumn{1}{|c|}{ Técnica de investigación } & \multicolumn{1}{|c|}{ Sujetos participantes } & \multicolumn{1}{c|}{ Cantidad } \\
\hline Grupo focal & Madres de familia entre 23 y 78 años. & 5 grupos focales \\
\hline Grupo focal & $\begin{array}{l}\text { Líderes comunitarios: fundadores, } \\
\text { integrantes del Consejo Comunitario } \\
\text { de Desarrollo, maestros de la escuela, } \\
\text { líderes religiosos. }\end{array}$ & 5 grupos focales \\
\hline Historias de vida & $\begin{array}{l}\text { Mujeres entre 35 y } 55 \text { años que fueron } \\
\text { casadas siendo menores de edad entre } \\
11 \text { y 15 años. }\end{array}$ & 5 historias de vida \\
\hline Historias de vida & $\begin{array}{l}\text { Hombres que fueron casados con una } \\
\text { mujer menor de edad. }\end{array}$ & 2 historias de vida \\
\hline Entrevista a profundidad & $\begin{array}{l}\text { Padres de familia, madres de familia, } \\
\text { comadronas, líderes comunitarios, } \\
\text { funcionarios del servicio de salud, } \\
\text { epresentante del RENAP. }\end{array}$ & 22 entrevistas a profundidad \\
\hline
\end{tabular}

\footnotetext{
a Population Council implementa el Programa Abriendo Oportunidades ${ }^{\circledast}$ en Chisec, Alta Verapaz desde el año 2013 creando grupos de niñas y adolescentes a nivel comunitario en alianza con los COCODES y las familias. A la fecha ha trabajado en 50 comunidades del municipio, formado a 35 mentoras comunitarias y atendido a más de 2000 niñas, adolescentes y jóvenes. El Q'eqchi' es el idioma materno de la mayoría de las y los participantes de investigación, por lo que la recolección de datos se realizó en este idioma.

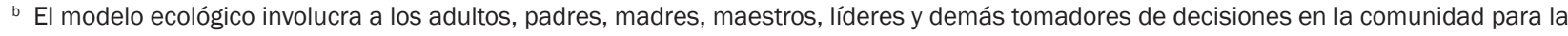
promoción de la causa del empoderamiento de las niñas como una estrategia que impacta en el desarrollo de toda la comunidad.

c La traducción al español fue realizada por el Lic. Arturo Chub, antropólogo q'eqchi' con certificación en la traducción español-q'eqchi'. La sistematización y traducción de la información se validó junto al equipo de investigación de campo para cuidar el sentido original de lo recopilado.
} 
Se analizaron los discursos recopilados para identificar las percepciones, normas y prácticas que emanan de las instituciones sociales alrededor del M/UIF. El director del Registro Nacional de las Personas -RENAP- al enterarse de la investigación, solicitó al equipo de recolección de datos comparar los registros de inscripción de nacimientos antes y después de la aprobación del Decreto 8-2015.

Por consideraciones éticas y de seguridad se decidió no incluir en la investigación a jóvenes menores de edad que hubieran sido casadas o unidas, por lo que no se cuenta con el aporte de sus experiencias y percepciones de este sector de población. La totalidad de participantes fueron mayores de edad. 


\section{Resultados}

Los resultados a continuación están organizados según la institución social a la que se refieren. Para cada una se ilustra como cada uno de los tres modelos se manifiesta...

Esta investigación revela que, en las comunidades investigadas, a pesar de la prohibición del matrimonio entre/con menores de edad las uniones infantiles forzadas continúan vigentes en la práctica. Se evidencia que las y los actores de todas las instituciones sociales conocen los cambios en la ley, y las consecuencias perjudiciales que tienen las UIF sobre la salud y la vida de las niñas y adolescentes. Describe prácticas y normativas que se dan entre las familias y la religión para reforzar la discriminación hacia la mujer. Evidencia la gran ausencia del gobierno y la educación como actores clave para hacer cumplir la ley y fortalecer la agencia de las niñas y adolescentes. A través de las historias de vida es posible identificar condiciones que suelen estar presentes alrededor de las UIF como la pobreza, la violencia y la falta de redes sociales. Recopila la percepción que tienen adultos y adultas desde su rol en las diversas instituciones sociales sobre el incremento intergeneracional que tienen las adolescentes hoy en el acceso a la tecnología, la educación y la socialización. A continuación, se detallan los hallazgos relativos a las distintas instituciones sociales estudiadas.

La familia: Como proceso de socialización, la educación trasciende el ámbito escolar. En este sentido se evidencia que, tanto la familia como la religión, son instituciones que pueden modificar las normas sociales referentes al UIF. Entre las respuestas se muestra el inicio del cambio en sus percepciones y discursos sobre este fenómeno. En este sentido una mujer adulta que fue casada siendo mejor de 15 años comentó: "Hoy, yo digo que no es bueno que las niñas se casen porque es una responsabilidad muy grande. Yo aconsejo a mis hijas que se cuiden y planifiquen, porque ahora hay información en el centro de salud" (Historia de Vida. Mujer adulta casada siendo menor de edad). Integrantes de la comunidad manifiestan conocer las razones por las que se ha retrasado la edad del matrimonio, por ejemplo, comentó una madre participante en un grupo focal que: "Es necesario que se casen hasta que tengas su DPI -documento de identificación- a los 18 años. Es para que disfruten su juventud y que puedan seguir estudian-
Las historias de vida de mujeres adultas que sufrieron el M/UIF permitieron identificar su efecto sobre la trayectoria de vida. Presentan un punto de referencia para la identificación de modificaciones que se han presentado de forma paulatina en el contexto en que se da el M/UIF. Este abordaje es útil para ilustrar las inequidades tanto a través de la perspectiva de género como generaciones.

do en una escuela" (Grupo focal. Madre de 23 años). Sin embargo, las acciones familiares y religiosas en torno a las adolescentes embarazadas documentan continuidad en los procesos de exclusión y condiciones que fuerzan a la unión de la pareja. Tal es el ejemplo de un padre que argumenta que si su hija no acepta casarse "es porque solo quiere divertirse con cualquier hombre y quiere estar con varios hombres. Si solo se une quiere decir que no es seguro y que quiere tener otro marido, porque no le gustó el que la está pidiendo" (Entrevista. Padre de familia).

La pobreza de la familia también promueve en las niñas el interés de salir de casa. Abundan los comentarios como el siguiente que reflejan la condición de pobreza como una explicación aceptable a la realización de la unión a temprana edad: "Lo que comíamos era chile con agua pura y es por eso por lo que decidí juntarme, porque veía la necesidad de nuestra familia" (Grupo focal. Mujer de 23 años). Se considera que, a través de la constitución de una nueva familia, la adolescente podrá optar a una mejor condición económica a la vez que descarga a su propia familia del costo de su manutención. "Por esa misma época tuve un noviazgo que duró un año y luego me junté con él. Sus padres llegaron a pedirme y los míos no querían aceptar, pero yo sí deseaba casarme. Yo pensaba que el matrimonio era lo mejor para mis papás porque como éramos bastantes hermanos, así serían menos los gastos, y además yo quería tener mis propias cosas" (Historia de vida. Mujer adulta casada siendo menor de edad).

Se registró que los UIF responden a la norma que determina como responsabilidad de padres y madres asegurar que sus hijas se casen como un mecanismo para mantener la honorabilidad de la familia aplicada en resguardo de la 
sexualidad de la adolescente. Las respuestas de los grupos focales confirmaron que el embarazo es una condición que demanda la unión y el matrimonio. "Si está embarazada, nosotros los padres, las obligamos a unirse o casarse para que no nos den vergüenza al tener un hijo sin papá” (Grupo focal. Secretario de COCODE).

Si bien se mencionaron múltiples consecuencias de las UIF, destacan las vinculadas con la violencia. La mayoría de las historias de vida de mujeres adultas que fueron casadas o unidas siendo menores de edad, dan cuenta de la violencia física y emocional que sufrieron desde niñas y su continuidad en el matrimonio de esta hasta la adultez. En estos casos, la violencia hacia las niñas era infligida por el padre y en algunos casos por la madre, en la escuela o camino a ella ya sea por los docentes, su pareja, hombres de la comunidad o estudiantes. Las motivaciones para infringirla eran diversas; en el hogar tenían que ver con su labor en el hogar o el alcoholismo. Una vez conformada la nueva pareja, las niñas y adolescentes confrontan la violencia en este nuevo espacio doméstico viéndose forzadas a asumir el rol subordinado que se les asigna por ser mujeres. Al respecto de las niñas y adolescentes unidas, explicaba una mujer que "Ellas sufren mucho porque no saben su trabajo. Desde alli empieza la violencia física y no saben cuidar a sus hijas e hijos" (Grupo focal. Mujer de 33 años).

La presión social para que la niña cumpla con el rol de género esperado se ejerce a través de la violencia física y emocional como un continuum desde su infancia que en casos se extiende a la vida adulta. Explica un facilitador comunitario sobre este aspecto: "Cuando piden a una niña de 13 para casamiento o para unirse con una persona mayor que ella o con uno de la misma edad, (...) la suegra la empieza a mandar a hacer las cosas de la casa, peor si la muchacha no puede trabajar bien es alli donde empiezan los problemas el hombre la empieza a insultar porque la mama le dice en dónde te sacaste a esta mujer no sirve para nada, y cuando la muchacha está embarazada le empieza a doler la cabeza, los nervios más cuando el hombre la insulta, la patea, o le pega a cada rato."

Algunos de los patrones identificados respecto al rol de la familia en relación con una UIF, incluyen roles específicos que cada integrante de la familia juega. Mientras el padre de familia decide cómo será el matrimonio, la madre es la encargada de hablar con la niña y convencerla. Los hermanos hombres presionan y aconsejan a la niña para que obedezcan a sus papás. Los abuelos acompañan a los
"Si yo quebraba un trasto, de castigo ella (la madre) me rajaba mi piel con uno de los pedazos. A las tres de la mañana había que levantarse para moler el maíz y si no me despertaba, me echaba agua para que me pusiera en pie. Me decian que tenía que aprender para poder atender a mi marido. Mi papá decía que por ser mujeres nos teníamos que casar porque el hombre nos iba a mantener y que lo único que íbamos a hacer era cuidarlo a él y a nuestros hijos."

Historia de vida. Mujer unida siendo menor de edad.

padres durante la pedida. La niña, obedece. En este marco se entiende que padre y madre siguen ejerciendo el control sobre la vida de la hija y tienen la autoridad para ejercer sanciones coercitivas en su contra.

Además de las uniones impuestas por los padres de familia, se presentaron casos en los que son las mismas adolescentes quienes optan por unirse. Esto supone surgir del deseo de una mejor vida -económica y afectiva- que la que tienen las jóvenes con su familia. Algunos participantes argumentaron que esto es posible como consecuencia de un incremento en el ámbito de socialización que tienen las adolescentes en la actualidad -vía los teléfonos, la tecnología, las redes sociales y la escuela- "Así como crecimos no había celular y no nos comunicábamos y no conocíamos qué era un celular. Ahora las niñas de 12 años tienen celular y solo con eso el muchacho llama que necesita hacer un encuentro en algún lugar" (Grupo focal. Mujer de 31 años). La comunidad percibe que hay una progresiva modificación de la dinámica familiar en la que la autoridad del padre y de la madre sucumbe frente a una supuesta autonomía asumida por las hijas. Se demuestra en el siguiente comentario: “El papá y mamá de una niña es el celular y lo que hacen los padres te van avisar pero ya solo porque se quedan en vergüenza, y si ya está embarazada ya es obligatorio que se case" (Grupo focal. Mujer de 50 años). Esta situación amenaza, según la comunidad, la integridad de adolescentes pues ya no es posible darles consejos ni determinar qué sucede con su vida. Al ser así, los padres y madres comentan que sienten que se dificulta su posibilidad de proteger a su hija ante una pareja que no ha sido seleccionada por ellos. "Hay muchos problemas porque si se enamoran en la calle, en la escuela o en algún otro lugar cuando se juntan o se casan hay muchos problemas porque no hay alguien quien pregunte por ella" (Grupo focal. Mujer de 52 años). 
"Son los padres de familia que deciden si una niña se va a juntar o casar (...) hay jóvenes que ya tienen planificado casarse...ahí ya no se puede hacer nada, pero para mí eso es una mentira ya después se separan."

\section{Facilitador comunitario 1, entrevista}

La religión: La comunidad menciona como actores importantes en la religión a pastores, sacerdotes, ancianos de la iglesia, ministros de la palabra, grupos femeninos y mayordomos. A pesar de que en el área de Chisec se practica la espiritualidad maya, sus actores no figuraron con relevancia dentro de los diálogos que se sostuvieron.

En la información recopilada se constata que las iglesias no avalan los matrimonios ni las uniones entre menores de edad, pero tampoco existe alarma o denuncia por ello. Si bien las uniones anteceden en muchos casos al matrimonio, es el ritual religioso el que legitima la unión de la pareja sin importar la edad.

La figura del pastor y del sacerdote juega un papel importante en el ritual de la pedida y siempre dan consejo de la Biblia y los deberes del hombre y la mujer. También refuerzan los roles tradicionales de género como el orden familiar aceptado moralmente. Esta autoridad religiosa acompaña cuando las familias ya han acordado la entrega de la niña y su bendición significa la aceptación moral de la unión. A través del ritual del matrimonio se legitima la unión de la pareja. En la mayoría de los casos se da muchos años después en que se da la unión. "La boda venía después, podía tardar un buen tiempo y el matrimonio se formalizaba en la iglesia." (Historia de Vida. Hombre de 70 años). Como sanción social, se descalifica la moral de quienes son madres solteras o estuvieron viviendo en pareja y luego se separaron. La religión desarrolla un proceso de exclusión social hacia ellas y sus hijos si se oponen al casamiento o si son madres solteras a través de: (1) la exclusión de participar en cargos dentro de la Iglesia; (2) negación de bautizo para sus hijos; (3) condenación al infierno por vivir en pecado y (4) promoción de la vergüenza y humillación ante la comunidad. Una mujer de 39 años comenta que "Cuando una niña se queda embarazada hay que dar a conocer a Ios COCODES y la comadrona. Ellos dicen que es necesario que se casen y cuando quieren entregan a los hijos en la Iglesia, cuando no están casados no se puede bautizar a un niño." (Grupo focal. Mujer de 39 años).
La economía: Los datos socioeconómicos recabados entre los y las participantes en las cinco comunidades revelan que se dedican principalmente al comercio y la agricultura. Los ingresos mensuales familiares varían entre los Q.600.00 a Q.2,000.00 (equivalente en US Dólares a \$82 a \$274). Este monto es insuficiente para la manutención de familias de 6 a 10 integrantes cuando la canasta básica es de Q.3,697 ${ }^{11}$. Las limitaciones para acceder a tierra y trabajarla, el desempleo y la fluctuación en los precios de los cultivos son condiciones que rodean el entorno de la niña y la exponen a pobreza, migración y violencia. En este contexto se reporta que las familias recurren al UIF como un medio para la distribución de la carga económico -el costo de manutención de la hija- y la adquisición de un mecanismo de producción -el trabajo del yerno-.

En la actualidad se mantienen tres condiciones de vida: la pobreza, la violencia y la discriminación hacia la mujer. La mayoría de las mujeres consultadas afirman que no son originarias de las comunidades donde actualmente viven. sus padres y madres vivían en terrenos alquilados o fincas donde trabajaban como mozos por lo que el acceso a tierra estaba condicionado al préstamo de mano de obra bajo condiciones y acceso a servicios precarios. También hacen mención de que las condiciones de pobreza como una limitante para acceder a la educación, servicios de salud y alimentación. "En la hacienda había un riachuelo, ahí lavábamos ropa, los trastes, el nixtamal y también allí nos bañábamos porque no teníamos chorro ni pila" (Historia de vida. Mujer 58 años).

En las entrevistas realizadas, se mencionó que, si bien es importante que el hombre que quiera casarse debe tener casa, trabajo y tierra para mantener a la familia, en la realidad no siempre sucede así. Los que no tienen esos recursos deben "convencer" al padre de familia de que tienen capacidad de proveer bienestar a la niña mediante el regalo de su trabajo a la familia. Así narró su experiencia una mujer que fue casada antes de los 15 años: “Decidieron que sería mi esposo porque había dado su mano de obra a cambio. Yo no lo podía creer" (Historia de vida. Mujer unida a los 13 años). Es así como se llega a negociar el matrimonio.

Además del imperativo moral de guardar el honor de las niñas, el matrimonio y unión temprana forzada significa la transferencia de la niña a cambio de un pago económico en trabajo o bienes. Las mujeres consultadas afirman que una de las razones para aceptar ser casadas era la pobreza 
en el hogar. Casándose o uniéndose ayudarían a que su familia no se preocupara más por alimentarla y vestirla. Se confirma que esta norma sigue vigente en la actualidad, por los testimonios de los grupos focales mixtos donde indican que las familias con recursos escasos aceptan rápidamente entregar a sus hijas para solventar deudas o por la incapacidad para mantener a sus hijos. Una participante manifestaba que: "Hay familias que no tienen suficientes recursos económicos y tiene muchos hijos y llega alguien a pedir a alguna hija, los padres aceptan inmediatamente para que ellos ya no tengan muchos hijos que mantener $y$ los hermanos felices que alguien de la familia se valla de la casa”. (Grupo focal. Mujer de 36 años).

La educación: Las historias de vida revelan que una de las limitaciones para continuar con sus estudios radica en el acoso ejercido por sus compañeros y por docentes, tanto dentro como fuera de la escuela. Esto fue una de las causas por las cuales los padres las retiraban de la escuela. En tal sentido el testimonio de una mujer quien finalmente fue casada forzosamente: "Cuando tenía 12 años empecé a estudiar, pero por la distancia que había entre mi casa y la escuela, dejé de ir. Además, cuando salía de clases, a veces en la calle me encontraba a muchachos que me abrazaban y eso no me gustaba, por esa razón sólo fui un mes. Tal vez hubiera podido seguir, pero mi mamá me sacó". (Historia de Vida. Mujer 34 años).

A lo largo del estudio no se reportó que la escuela realizara acciones afirmativas por detener la deserción escolar de las niñas y adolescentes; o acciones de prevención al acoso sexual o de generación de entornos seguros. Tampoco se mencionaron acciones afirmativas -por parte de la escuela, la familia ni la comunidad- que dirigieran la atención a la eliminación del acoso y la garantía del derecho de las niñas. Al abordar el vínculo entre educación y UIF, lejos de identificar en la escuela un aliado, la comunidad se refiera a ella como una amenaza por ser un espacio de socialización sin supervisión de la familia, que además para muchas madres es desconocido al no haber ellas mismas asistido a la escuela. La educación escolarizada es limitada y no refiere acciones contundentes que incrementen la asistencia de las adolescentes a partir de la pubertad. En su contenido curricular, así como en sus prácticas cotidianas, actúa en aceptación a la normativa tradicional y legitima la violencia hacia las niñas y adolescentes.

\section{Del Gobierno Central Gobierno Comunitario: El Estado} reconoce que las UIF son un problema que obstaculiza el
“Ahora hay una ley, pero las personas y los jóvenes no

entienden y no creen la ley no podemos hacer nada si

se juntan, la ley no sabe nada de lo que está pasando,

lo están haciendo a escondidas, ahora la ley prohíbe

el matrimonio. de la niñez por ejemplo si yo quisiera

casar a una de mis hijas de 12 años no me reciben

sobre la mesa una secretaria, es muy dura la ley de

ahora."

Testimonio durante grupo focal

futuro de las niñas. Además, el Estado de Guatemala ha ratificado tratados y convenios internacionales relativos a los derechos de la niñez y la juventud. A pesar de que existen sanciones legales, no se evidenció en la investigación que éste asuma acciones contundentes y sostenidas para erradicar las UIF. Desde que existe la modificación al Código Civil 13-2017 ésta ha sido evadida y no hay ningún seguimiento de parte de las autoridades. Se aduce a la falta de recursos o de capacidades.

En las comunidades estudiadas se reconoce la autoridad legítima de los Comités Comunitarios de Desarrollo -COCODE-. Estos comités son un producto del Decreto de Ley 11-2002 cuyo objetivo es promover la participación de los miembros de una comunidad en la gestión pública para llevar a cabo el proceso de planificación democrática del desarrollo.

Cuando el decreto 8-2015 entró en vigor se la municipalidad realizó acciones para darla a conocer a nivel comunitario. Se convocó a los COCODES para informar sobre la misma y las sanciones que se aplicarían a quienes rompieran la norma al autorizar matrimonios en su comunidad. De la misma forma se realizaron reuniones informativas convocadas por el Centro de Salud para informar a las comadronas para que refieran a las menores de edad embarazadas en parto a los hospitales. La medida ha sido útil para informar y provocar conocimiento sobre el daño que hace a las niñas y adolescentes las uniones y embarazos tempranos. La población reconoce que el matrimonio entre/con menores tendrá como sanción la cárcel o multa y que se considera delito el intercambio económico en la negociación del matrimonio (trata de personas).

Sin embargo, la incapacidad de hacer valer los derechos y aplicar la sanción establecida deja en vulnerabilidad a la niñez de la región, las uniones de hecho continúan siendo 
practicas recurrentes que simplemente ya no son registrados bajo un acuerdo matrimonial. Se documentó el uso de estrategias usadas para evadir el Decreto 08-2015 como actas notariales de matrimonios entre/con menores de edad que registraron fechas de inscripción previo a la fecha de emisión del decreto y la inscripción de recién nacidos/as como hijos de sus abuelos. Con el nuevo decreto, el RENAP está en la obligación de denunciar como delito las uniones que involucren a menores de edad. De los 223 expedientes de nacimientos registrados en el RENAP, durante el período de la investigación, se identificaron seis casos de embarazos en niñas (entre 11 a 13 años) que fueron notificados ante el MP, los expedientes de adolescentes de 14 a 17 años no fueron denunciados. Aunque el RENAP denuncia ante el MP las uniones en menores, no hay seguimiento por diferentes razones (no hay recursos para pagar abogados, la comunidad se opone, etc.). Posterior a la aprobación del decreto 8-2015, en Chisec los nacimientos registrados por RENAP indican que el 59.19\% de las madres tenían entre 14 a 17 años; dato que contrasta con la edad registrada de los padres pues el $44.84 \%$ tienen 25 años. Si bien ya no se registran los matrimonios se continúan las uniones entre niñas adolescentes con hombres mayores de edad. 


\section{Discusión de Resultados}

Esta investigación es un estudio de caso cuyos resultados se aplican únicamente a las comunidades participantes en este particular período de tiempo. Por la metodología utilizada, la muestra es pequeña y su integración varió entre las comunidades por la respuesta a la convocatoria. Dada la sensibilidad del tema, las historias de vida se realizaron con mujeres adultas que fueron casadas/unidas antes de los quince años.

Los resultados manifiestan que se presenta una transición -en diferente grado- hacia la eliminación del M/UIF en las instituciones sociales. En tal sentido se pueden tipificar tres formas en que se dan los M/UIF: (1) Como, en el pasado, los padres arreglan los matrimonios y uniones de las hijas sin que ellas pudieran opinar nada; (2) Los padres y madres arreglan los matrimonios o uniones para salvar la moral familiar y ocultar un embarazo; y (3) los matrimonios y uniones son producto de la decididos de las jóvenes. En el primer caso, a ser los padres y madres los responsables de la unión, se reconoce su derecho a intervenir cuando identifican que algo dentro de la relación no está bien para su hija. En caso de que las jóvenes deciden por sí mismas casarse, se establece como norma que ni su padre ni su madre pueden intervenir en caso de identificar algo nocivo en la relación.

A lo largo de la investigación fue evidente la sanción hacia las adolescentes cuando están estudiando o van a estudiar a nivel básico o diversificado; pues en esa etapa coincide la pubertad y la adolescencia con el mito de que las niñas están aptas para el matrimonio o unión. La escuela, como institución que reproduce la normativa social, también traslada el tabú y el silencio alrededor de la educación en sexualidad. Aunque ha habido esfuerzos para informar a los centros de salud, la información brindada no es objetiva y está permeada de valores religiosos. Actualmente, con la globalización, las telecomunicaciones y la moda, existe una exposición de la juventud a la información sin restricciones que no está recibiendo acompañamiento científico por parte de los adultos y la escuela en materia de derechos sexuales y derechos reproductivos.

Las entrevistas y grupos focales enfatizan un aumento de uniones formadas sin la intervención de los padres y las familias. Las madres y padres aducen que esto está sucediendo por el acceso a la escuela y a la tecnología como espacios en los que sostienen relaciones con hombres sin la supervisión ni consentimiento de su familia. Lejos de perfilar el sistema educativo como un factor protector, éste constituye, a los ojos de los adultos de la familia, como un riesgo para las niñas. La falta de acciones de las escuelas para proteger a las niñas del acoso y la discriminación es un ejemplo de cómo el Estado -a través de sus instituciones- permanece como un sujeto prácticamente ausente con excepción del impulso de la legislación y la negativa a inscribir matrimonios que contradicen el marco legal.

La literatura sobre el M/UIF argumenta que socava la capacidad de las niñas y adolescentes para acceder a una educación que permita desarrollar su autonomía y las expone a las consecuencias peligrosas del parto antes de la madurez física ${ }^{17}$. Los resultados de esta investigación confirman, además, que las familias y comunidades están conscientes de este hecho.

Las comunidades estudiadas cumplen con el perfil de la prevalencia del M/UIF en áreas rurales, remotas y en condiciones de pobreza ${ }^{6} \mathrm{y}$ al no haber cambio en esta condición, sigue vigente el UIF como una estrategia de sobrevivencia tanto para las familias como para las niñas y adolescentes.

Los resultados destacan que las generaciones de mujeres casadas antes de los 15 años no tuvieron acceso a educació $n^{16}$. Los testimonios de las familias en los grupos focales indican que, el acceso a la escuela facilita las UIF y que las niñas adolescentes ahora deciden por ellas mismas cuándo y con quién unirse. Si bien la UNESCO estipula que una a educación eficaz, basada en el desarrollo de competencias, conciencia acerca de los riesgos vinculados con el sexo sin protección y el uso de drogas, fomenta la adopción de comportamientos más sanos, y desarrolla actitudes y valores que promueven los derechos humanos y la igualdad de género ${ }^{30}$, esta no es la experiencia educativa de las adolescentes y jóvenes en las comunidades participantes. El limitado acceso a la educación, la reproducción de normas de género discriminatorias y la omisión de acción son variables importantes para comprender el papel de la 
Escuela en los matrimonios y uniones tempranas forzadas en las comunidades investigadas. Al respecto Haberland ha indicado que la propuesta de proveer educación integral en sexualidad, cuando se implementa con perspectiva de género, incide en una disminución de la violencia y conductas de alto riesgo ${ }^{9}$.

El Decreto 13-2017 emitido por el Congreso de la República de Guatemala es el primer paso para la erradicación del M/UIF, aunque las dudas sobre su incidencia permanecen vigentes en las comunidades estudiadas; coincidiendo con otros estudios ${ }^{28}$. Como institución social, la familia dicta las normas y prácticas vinculadas a las formas de parentesco y reproducción que permitirán el sostenimiento del grupo social ${ }^{10}$. Para el caso de las comunidades estudiadas, los cambios legales no son suficientes para provocar el cambio en las normas de género que otorgan mayor valor a los niños y hombres que a las mujeres ${ }^{16}$. Los COCODES y COMUDES dependen de los recursos y de la voluntad que los administradores públicos locales y nacionales tengan de canalizar respuestas adecuadas a las necesidades planteadas por los pobladores. El control de los consejos nacionales, regionales y departamentales radica en autoridades del gobierno ejecutivo quienes priorizan en base a intereses partidarios o electorales, anulando el espíritu de descentralización. Esto vulnera a los líderes comunitarios de los COCODES, convirtiéndolos en mediadores entre un Estado débil y desinteresado en la población y una población que desconoce sus derechos y las responsabilidades del Estado. 


\section{Conclusiones y Recomendaciones}

Los resultados de esta investigación pueden ser utilizados en el diseño de acciones comunitarias que buscan provocar una disrupción social en las normas, percepciones y acciones que, desde las diferentes instituciones sociales, inciden en la erradicación del UIF. Este fue el caso práctico para el diseño de la campaña radial liderada por Population Council en el municipio de Chisec durante el último trimestre del 2017.

La Teoría de Cambio del Population Council sostiene que la base de una vida productiva se forma durante la adolescencia, junto a las habilidades para la vida cotidiana, los conocimientos sobre finanzas, la salud sexual y reproductiva, la capacidad de negociación y la alfabetización. También es el período durante el cual la pobreza se puede consolidar o superar ${ }^{25}$. En el momento en que las niñas y adolescentes vulnerables enfrentan presiones sociales para alentar o imponer las UIF, es esencial que tengan las habilidades para negociar y manejar las desigualdades de poder dentro de la familia, los pares y las autoridades comunitarias 6 . Al alcanzar la pubertad, las niñas casadas o en unión necesitan una red social de amigos de confianza, para desarrollarse y mantener la agencia requieren asistencia de otros y estrechan lazos de afiliación ${ }^{8}$.

Diseminación de información: Es determinante difundir información sobre los efectos nocivos de las UIF entre padres de familia, autoridades y adolescentes. La información puede diseminarse a través de estrategias informativas que les sean familiares y permitan identificarse con la evidencia. Es necesario trascender la información de las estadísticas para alcanzar la conciencia que genera el conocimiento y análisis de casos que son familiares o con los que encuentran similitudes. Asimismo, es necesario evidenciar los beneficios económicos que se producen cuando las adolescentes consolidan su agencia, aumentan los años de estudio y se desarrollan en entornos seguros.

Se hace necesario analizar la institución social de la Economía desde dos perspectivas: las condiciones materiales que generan vulnerabilidad en la niña condicionándola al matrimonio como mecanismo de supervivencia y la economía sexual donde la niña y la mujer tienen un papel oculto en la producción y como objeto de intercambio.
Es necesario sensibilizar a los COCODES y COMUDES y socializar las rutas de denuncia para que trasladen los casos de UIF en sus comunidades al sistema judicial municipal. También hay que fortalecer a las instituciones de justicia -municipal y departamental- con personal capacitado y con recursos suficientes para atender la demanda de forma adecuada. Sugerimos la priorización de (1) la creación de condiciones para la transición segura de las niñez a la adulta -10 a 19 años- reconociendo que este período de la vida es crucial para su fortalecimiento y desenvolvimiento en la vida adulta; (2) el reconocimiento de las normas y sanciones sociales que son discriminatorias para las niñas; (3) la ampliación del espacio de participación de las niñas, garantizar que tenga la oportunidad de acceder a estos espacios y desenvolverse de forma libre y segura en ellos (escuela, participación comunitaria, desarrollo económico) y (4) el reconocimiento que las adolescentes que ya son madres necesitan apoyo e inversión, tanta como las que aún no están casadas 10 citando a Mensch et al. (1988).

Fortalecimiento de acciones desde el Estado: Es necesaria la promoción de acciones que a nivel estatal y comunitario que aseguren entornos seguros para las niñas Q'eqchi's en las escuelas, facilitación de información objetiva y veraz sobre los derechos y sexuales en los centros de salud y comadronas.

Provisión de educación integral en sexualidad: Las escuelas tienen que asumir evitar y sancionar el acoso de los hombres hacia las mujeres e incorporar la educación integral en sexualidad que incluye relaciones de poder y dinámicas de género ${ }^{\text {. }}$.

Para superar las condiciones de discriminación de género, algunas metas que podría adoptar la educación es la provisión de herramientas que equipen a las estudiantes con conocimientos para ser adultas productivas en la sociedad. Es importante que desde el aula se fomente la habilidad de contribuir al bienestar colectivo como ciudadana e integrante de la comunidad a la que se pertenece y fortalecer las capacidades para tomar decisiones basadas en la identidad propia, la integridad y el bienestar ${ }^{10}$.

Es necesario que la escuela aborde la interpretación de los adultos sobre la asisteencia a la escuela como causa de uniones tempranas decididas entre los estudiantes. 


\section{Referencias}

1. Amin, S., Ahmed, J., Saha, J., Hossain, I., \& Haque, E. (2015). Delaying child marriage through community-based skills-development programs for girls: Results from a randomized control trial in rural Bangladesh. New York: Population Council.

2. Chae, S. a. (2017). The Global State of Evidence on Interventions to Prevent Child Marriage. GIRL Center Research Brief No.1. New York: Population Council.

3. Congreso de la República. (2015). Decreto 8-2015 Código Civil. Guatemala.

4. Congreso de la República de Guatemala. (2017). Decreto 13-2017 Código Civil. Guatemala.

5. Durkheim, É. (1998). Educación y Sociología. México: Editorial Coyoacán.

6. Erulkar, A. (2013). Adolescence Lost: The realities of child marriage. Journal of adolescent health, 513-514.

7. Gauer, M., \& Tanner, S. (2018). The Missing Target Changing Beliefs and Behaviors to Deliver Gender Equality. Plan Internacional.

8. Guidry-Grimes, L., \& Victor, E. (2012). Vulnerabilities compounded by social institutions. International Journal of Feminist Approaches to Bioethics, 5(2). University of Toronto Press, 126-146.

9. Haberland, N. A. (2015). The Case for Addressing Gender and Power in Sexuality and HIV Education: A Comprehensive Review. International Perspectives on Sexual and Reproductive Health, 31-42.

10. Harper, C., Jones, N., \& Watson, C. (2012). Gender justice for adolescent girls: tackling social institutions. London: Overseas Development Institute.

11. Instituto Nacional de Estadística INE. (2017). Índice de Precios al Consumidor y Costo de la Canasta Básica Alimentaria y Vital 2016. Guatemala.

12. International Justice Mission. (2013). Guatemalan justice system performance study 2008-2012: indicators of practice, process and resolution within cases of child sexual assault.

13. Jensen, R. (2012). Do labor market opportunities affect young women's work and family decisions? Experimental evidence from India. The Quarterly Journal of Economics 127(2), 753-792.
14. Kolev, A., Nowacka, K., \& Gaëlle, F. (2014). Social Institutions and Gender Index.

15. Lee-Rife, S., Malhotra, A., Warner, A., \& Glinski, A. (2012). What works to prevent child marriage: A review of the evidence. Studies in Family Planning 43(4), 287-303.

16. Loaiza, E., \& Wong, S. (2012). Marrying Too Young: End Child Marriage. New York: United Nations Population Fund.

17. Maswikwa, B. R. (2015). Minimum Marriage Age Laws and the Prevalence of Child Marriage and Adolescent Birth. International Perspectives on Sexual and Reproductive Health, Vol. 41, No.2.

18. Ministerio de Salud Pública y Asistencia Social, Instituto Nacional de Estadística, Centro de Control de Enfermedades. (2010). Encuesta Nacional de Salud Materno Infantil 2008-2009. Guatemala: Ministerio de Salud Pública y Asistencia Social.

19. Ministerio de Salud Pública y Asistencia Social, Instituto Nacional de Estadística, ICF International. (2017). Encuesta Nacional de Salud Materno Infantil 2014-2015. Ciudad de Guatemala. Guatemala: Ministerio de Salud Pública y Asistencia Social .

20. Naciones Unidas. (n.d.). Retrieved from http://www. un.org/esa/socdev/documents/youth/fact-sheets/ youth-definition.pdf

21. Naciones Unidas. (n.d.). http://www.un.org/esa/soc$\mathrm{dev/documents/youth/fact-sheets/youth-definition.pdf.}$

22. Ortega, J. A. (2011). Violencia escolar, resistencias y prácticas ciudadanas en Guatemala y Costa Rica. Guatemala: Universidad San Carlos de Guatemala.

23. Pérez, M. L. (2011). El matrimonio infantil y las uniones de hecho forzadas en adolescentes en Guatemala. Guatemala: UNICEF.

24. PNUD. (2016). Informe Nacional de Desarrollo Humano 2015/2016. Guatemala: Programa de las Naciones Unidas para el Desarrollo.

25. Population Council. (2011). Promoción de transiciones a la vida adulta sanas, seguras y productivas: La estrategia de Population Council. New York. 
26. Santhya, K., Ram, U., Acharya, R., Jejeebhoy, S., Ram, F., \& Singh, A. (2010). Associations between early marriage and young women's marital and reproductive health outcomes: Evidence from India. International Perspectives on Sexual and Reproductive Health 36(3), 132-139.

27. Singh, K., \& Kapur, D. (2001). Law, Violence and the Girl Child. Health and Human Rights, 8-29.

28. Svanemir. (2015). Research priorities on ending child marriage and supporting married girls. US National Library of Medicine. National Institutes of Health.

29. Swanemyr, J., \& Chandra-Mouli, V. (2016). Consistent Laws and Child Marriage. International Perspectives on Sexual and Reproductive Health, 218-221.

30. UNESCO. (2017). Estrategia de la UNESCO sobre la educación para la salud y el bienestar: contribución a Ios objetivos de desarrollo sostenible. París: UNESCO.

31. UNICEF. (2014). Ending Child Marriage: Progress and Prospects. New York: UNICEF.

32. Vaitla, B., Taylor, A., Van Horn, J., \& Cislaghi, B. (2017). Social norms and girls' well-being Linking theory to practice. Washington D.C.: Data $2 x$.

33. Women's Refugee Commission. (2016). A Girl No More: The Changing Norms of Child Marriage in Conflict. NY: Women's Refugee Commission. 


\section{Anexos}

\section{ANEXO 1 - HISTORIAS DE VIDA DE MUJERES ADULTAS QUE FUERON CASADAS O UNIDAS SIENDO MENORES DE 15 AÑOS}

Con el propósito de identificar posibles transformaciones en el M/UIF se realizaron historias de vida con mujeres adultas que habían sido casadas o unidas antes de cumplir los 15 años. Éstas permitieron conocer los cambios en la percepción de las mujeres sobre el M/UIF. Se han incluido las historias de vida como un recurso de gran riqueza para el análisis del M/UIF como un fenómeno social.

\section{ANEXO 2 - MOVILIDAD SOCIAL DE LAS MUJERES CASADAS O UNIDAS SIENDO MENORES}

\begin{tabular}{|c|c|c|}
\hline \multicolumn{2}{|l|}{ 1. Comunidad Las Promesas } & \multirow{3}{*}{ Tendencias } \\
\hline Niñez & Adultez & \\
\hline Origen & Residencia actual & \\
\hline $\begin{array}{l}\text { Mis padres eran originarios de las } \\
\text { Fincas La Constancia y Las Mercedes, } \\
\text { en Telemán, municipio de Panzós. }\end{array}$ & $\begin{array}{l}\text { Comunidad Las Promesas, municipio } \\
\text { de Chisec. }\end{array}$ & Migración interna \\
\hline \multicolumn{2}{|l|}{ Condiciones materiales del hogar } & \\
\hline $\begin{array}{l}\text { En la hacienda había un riachuelo, ahí } \\
\text { lavábamos ropa, los trastes, el nixtamal } \\
\text { y también allí nos bañábamos porque } \\
\text { no teníamos chorro ni pila. }\end{array}$ & $\begin{array}{l}\text { Mi esposo trabajaba como chofer de } \\
\text { un tractor en la misma hacienda donde } \\
\text { vivíamos. Alli fabricaban cal y nos } \\
\text { ayudábamos con la venta de la cosecha } \\
\text { de maíz. Con mi esposo construimos } \\
\text { una casa en la misma hacienda. }\end{array}$ & $\begin{array}{l}\text { Trabajo del esposo en haciendas, fincas } \\
\text { en labores agrícolas. }\end{array}$ \\
\hline \multicolumn{3}{|l|}{ Composición de la familia } \\
\hline No Responde NR & $\begin{array}{l}\text { Tuvimos un total de } 9 \text { hijos, de los } \\
\text { cuales } 4 \text { murieron. Uno de ellos murió a } \\
\text { los } 15 \text { años porque lo habían embru- } \\
\text { jado. Los otros } 3 \text { murieron momentos } \\
\text { después del parto. Dos de ellos eran } \\
\text { niños y una niña. }\end{array}$ & \\
\hline \multicolumn{2}{|l|}{ 2. Comunidad Monte Cristo } & \multirow{3}{*}{ Tendencias } \\
\hline Niñez & Adultez & \\
\hline Origen & Residencia actual & \\
\hline $\begin{array}{l}\text { Sobre el origen de mis padres, no sé } \\
\text { exactamente de donde eran, creó que } \\
\text { eran de Raxruhá. No recuerdo muy } \\
\text { bien, a lo lejos recuerdo que estuvimos } \\
\text { en El Palmar y luego fuimos a Secacao. } \\
\text { Allí fue donde crecimos. Nos cambiába- } \\
\text { mos de lugar porque era una hacienda. }\end{array}$ & $\begin{array}{l}\text { Comunidad Monte Cristo, municipio de } \\
\text { Chisec. }\end{array}$ & Migración interna, sin acceso a tierra. \\
\hline \multicolumn{2}{|l|}{ Condiciones materiales del hogar } & \\
\hline $\begin{array}{l}\text { En aquella época, el maíz valía } 75 \text { cen- } \\
\text { tavos el quintal. Nadie nos molestaba y } \\
\text { mi papá disfrutó trabajando la tierra. Mi } \\
\text { papá estaba cansado de estar de lugar } \\
\text { en lugar hasta que nos quedamos en la } \\
\text { comunidad Monte Cristo. }\end{array}$ & $\begin{array}{l}\text { Cuando tuve a mi segundo hijo me } \\
\text { di cuenta de que fue más difícil el } \\
\text { matrimonio. Tenía que lavar su ropa y } \\
\text { cuando mis hijos se enfermaban y no } \\
\text { teníamos pisto para curarlo. }\end{array}$ & $\begin{array}{l}\text { Condiciones precarias con limitación a } \\
\text { acceso a servicios. }\end{array}$ \\
\hline
\end{tabular}




\begin{tabular}{|c|c|c|}
\hline \multicolumn{2}{|l|}{ Composición de la familia } & \multirow[b]{2}{*}{$\begin{array}{l}\text { Familias numerosas sin planificación de } \\
\text { embarazos. Mortalidad infantil. }\end{array}$} \\
\hline $\begin{array}{l}\text { Nosotros somos cuatro hermanas y } \\
\text { un hermano, pero también tengo } 5 \\
\text { hermanos ya fallecidos. }\end{array}$ & $\begin{array}{l}\text { Tuve todos los hijos que Dios quiso que } \\
\text { tuviera. (...) Tuve hijos hasta donde mi } \\
\text { cuerpo lo permitió. Ya terminé, el más } \\
\text { pequeño de mis hijos es el que está } \\
\text { acompañándome ahora. }\end{array}$ & \\
\hline \multicolumn{2}{|l|}{ 3. Comunidad Nueva Babilonia } & \multirow{3}{*}{ Tendencia } \\
\hline Niñez & Adultez & \\
\hline Origen & Residencia actual & \\
\hline $\begin{array}{l}\text { Nací en Purulhá, Baja Verapaz en la } \\
\text { Aldea Muxonté. Mis padres son de la } \\
\text { misma Aldea }\end{array}$ & $\begin{array}{l}\text { Comunidad Nueva Babilonia, municipio } \\
\text { de Chisec. }\end{array}$ & Migración Interna \\
\hline \multicolumn{2}{|l|}{ Condiciones materiales del hogar } & \\
\hline $\begin{array}{l}\text { Mi niñez fue muy triste porque mis } \\
\text { padres vivieron en la pobreza, no tenían } \\
\text { casa, solo alquilaban y tampoco tenían } \\
\text { terreno, solo vivíamos del trabajo que le } \\
\text { daban sus vecinos. }\end{array}$ & $\begin{array}{l}\text { Para criar a mis hijos trabajaba en el } \\
\text { corte de café y vendía tamales. }\end{array}$ & $\begin{array}{l}\text { Condiciones de vida precarias con } \\
\text { limitación a acceso a servicios. }\end{array}$ \\
\hline \multicolumn{2}{|l|}{ 4. Comunidad Pozo Seco } & \multirow{3}{*}{ Tendencia } \\
\hline Niñez & Adultez & \\
\hline Origen & Residencia actual & \\
\hline $\begin{array}{l}\text { Nací en un lugar llamado Chinamá, en } \\
\text { el Municipio de Lanquín. Vivía con mi } \\
\text { madre en una hacienda con mi madre, } \\
\text { a mi padre nunca lo conocí. }\end{array}$ & $\begin{array}{l}\text { Comunidad Pozo Seco, municipio de } \\
\text { Chisec }\end{array}$ & Migración interna \\
\hline \multicolumn{2}{|l|}{ Condiciones materiales del hogar } & \\
\hline $\begin{array}{l}\text { Mi madre trabajaba en la hacienda a } \\
\text { cargo de la alimentación de todos los } \\
\text { trabajadores y yo siempre me quedaba } \\
\text { en casa cuidando todos los animales. }\end{array}$ & $\begin{array}{l}\text { No sabía qué hacer (al fallecer su } \\
\text { primer esposo) y no me quedó otra que } \\
\text { buscar trabajo y encontré uno en un } \\
\text { comedor. } \\
\text { Aprendí a hacer pan y también me ocu- } \\
\text { paba de los oficios de la casa. Ellos me } \\
\text { trataban como la abuela de ellos, aun- } \\
\text { que no teníamos ningún parentesco. }\end{array}$ & $\begin{array}{l}\text { Hogar monoparental. Las mujeres } \\
\text { proveían el sustento familiar. }\end{array}$ \\
\hline \multicolumn{2}{|l|}{ Composición de la familia } & \\
\hline 1 hija & $\begin{array}{l}\text { Con mi primer esposo tuve tres hijos. } \\
\text { Uno murió ahogado en el río. }\end{array}$ & Pocos hijos y mortalidad infantil \\
\hline \multicolumn{2}{|l|}{ 5. Barrio San Francisco } & \multirow{3}{*}{ Tendencia } \\
\hline Niñez & Adultez & \\
\hline $\begin{array}{r}\text { Origen } \\
\end{array}$ & Residencia actual & \\
\hline Yo nací en la Aldea Setzí & $\begin{array}{l}\text { Barrio San Francisco, municipio de } \\
\text { Chisec }\end{array}$ & Migración interna \\
\hline \multicolumn{2}{|l|}{ Condiciones materiales del hogar } & \\
\hline NR & $\begin{array}{l}\text { Vivimos con mi esposo } 20 \text { años con mi } \\
\text { papá y solo nos heredaron el terreno } \\
\text { donde vivimos. }\end{array}$ & \\
\hline \multicolumn{2}{|l|}{ Composición de la familia } & \\
\hline NR & $\begin{array}{l}\text { Tuve } 10 \text { hijas e hijos, } 7 \text { están vivos y } 3 \\
\text { se murieron en el parto. }\end{array}$ & \\
\hline
\end{tabular}




\section{ACELERAR LOS ESFUERZOS}

para avanzar en los derechos de las
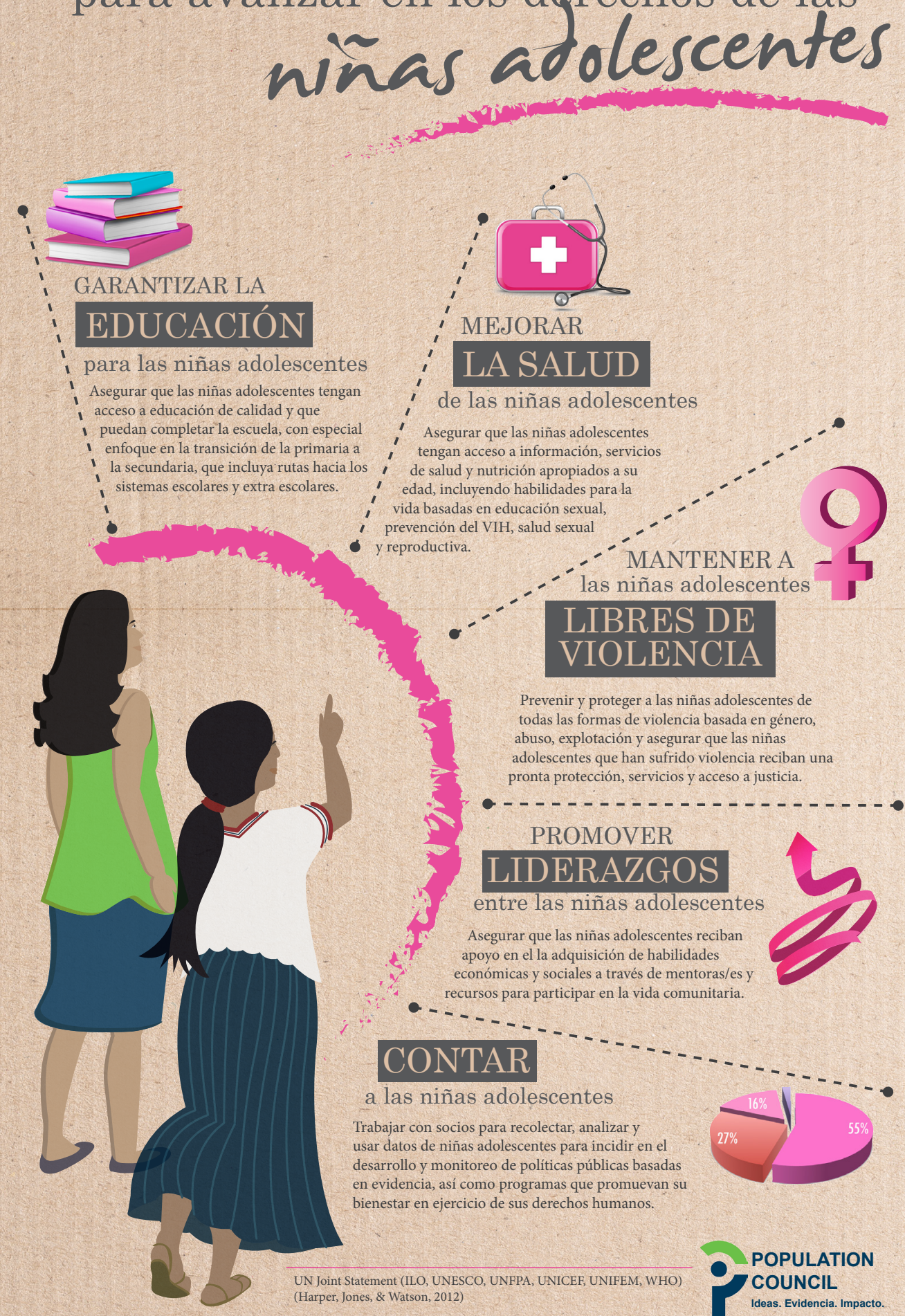
